\title{
Automated "cells-To-Peptides" Sample Preparation Workflow for High-Throughput, Quantitative Proteomic Assays of Microbes
}

Chen, Yan; Guenther, Joel M.; Gin, Jennifer W.; Chan, Leanne Jade G.; Costello, Zak; Ogorzalek, Tadeusz L.; Tran, Huu M.; Blake-Hedges, Jacquelyn M.; Keasling, Jay D.; Adams, Paul D.

Total number of authors:

13

Published in:

Journal of Proteome Research

Link to article, DOI:

10.1021/acs.jproteome.9b00455

Publication date:

2019

Document Version

Publisher's PDF, also known as Version of record

Link back to DTU Orbit

Citation (APA):

Chen, Y., Guenther, J. M., Gin, J. W., Chan, L. J. G., Costello, Z., Ogorzalek, T. L., Tran, H. M., Blake-Hedges, J. M., Keasling, J. D., Adams, P. D., García Martín, H., Hillson, N. J., \& Petzold, C. J. (2019). Automated "cellsTo-Peptides" Sample Preparation Workflow for High-Throughput, Quantitative Proteomic Assays of Microbes. Journal of Proteome Research, 18(10), 3752-3761. https://doi.org/10.1021/acs.jproteome.9b00455

\section{General rights}

Copyright and moral rights for the publications made accessible in the public portal are retained by the authors and/or other copyright owners and it is a condition of accessing publications that users recognise and abide by the legal requirements associated with these rights.

- Users may download and print one copy of any publication from the public portal for the purpose of private study or research.

- You may not further distribute the material or use it for any profit-making activity or commercial gain

- You may freely distribute the URL identifying the publication in the public portal 


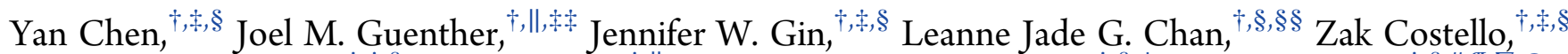
Tadeusz L. Ogorzalek, ${ }^{\dagger, t, \S}$ Huu M. Tran, ${ }^{\dagger}, \|$ Jacquelyn M. Blake-Hedges, ${ }^{\dagger, \S, \perp}$ Jay D. Keasling, ${ }^{\dagger}, \S, \#, \Psi, \nabla, O_{0}$ Paul D. Adams, ${ }^{\dagger, \#, ~ H e ́ c t o r ~ G a r c i ́ a ~ M a r t i ́ n, ~}{ }^{\dagger, \ddagger, \S, \dagger \dagger}$ Nathan J. Hillson, $^{\dagger, \ddagger, \S_{0}}$ and Christopher J. Petzold ${ }^{*, \dagger, \ddagger, \S}$

$\dagger$ Joint BioEnergy Institute and ${ }^{\ddagger}$ Agile BioFoundry, Lawrence Berkeley National Laboratory, Emeryville, California 94608, United States

${ }^{\S}$ Biological Systems and Engineering Division and Molecular Biophysics and Bioimaging, Lawrence Berkeley National Laboratory, Berkeley, California 94720-1460, United States

"Sandia National Laboratories (NTESS), Livermore, California 94551, United States

${ }^{\perp}$ Department of Chemistry and ${ }^{\#}$ Department of Bioengineering, University of California Berkeley, Berkeley, California $94720-1460$, United States

II Department of Chemical and Biomolecular Engineering, University of California, Berkeley, California 94720-1460, United States

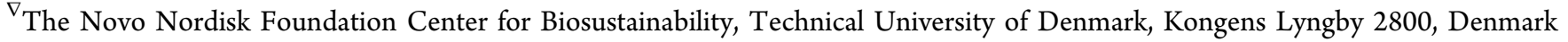

${ }^{\circ}$ Center for Synthetic Biochemistry, Synthetic Biology Institute, Shenzhen Institutes for Advanced Technologies, Shenzhen 518000, China

${ }^{\dagger \dagger}$ BCAM, Basque Center for Applied Mathematics, Bilbao, Bizkaia 48009, Spain

Supporting Information

ABSTRACT: Mass spectrometry-based quantitative proteomic analysis has proven valuable for clinical and biotechnology-related research and development. Improvements in sensitivity, resolution, and robustness of mass analyzers have also added value. However, manual sample preparation protocols are often a bottleneck for sample throughput and can lead to poor reproducibility, especially for applications where thousands of samples per month must be analyzed. To alleviate these issues, we developed a "cells-to-peptides" automated workflow for Gram-negative bacteria and fungi that includes cell lysis, protein precipitation, resuspension, quantification, normalization, and tryptic digestion. The workflow takes $2 \mathrm{~h}$ to

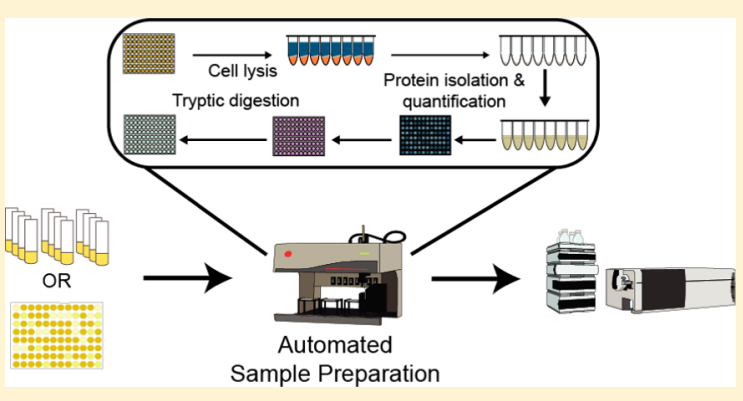
process 96 samples from cell pellets to the initiation of the tryptic digestion step and can process 384 samples in parallel. We measured the efficiency of protein extraction from various amounts of cell biomass and optimized the process for standard liquid chromatography-mass spectrometry systems. The automated workflow was tested by preparing 96 Escherichia coli samples and quantifying over 600 peptides that resulted in a median coefficient of variation of $15.8 \%$. Similar technical variance was observed for three other organisms as measured by highly multiplexed LC-MRM-MS acquisition methods. These results show that this automated sample preparation workflow provides robust, reproducible proteomic samples for high-throughput applications.

KEYWORDS: automation, sample preparation, proteomics, bacteria, fungi, microbes, biotechnology, high-throughput

\section{INTRODUCTION}

Quantitative proteomic assays are a key component of numerous clinical and biotechnology research studies. They inform biomarker discovery research, ${ }^{1-3}$ guide optimization of metabolically engineered organisms, ${ }^{4}$ and help characterize the protein structure and function. ${ }^{5}$ As such, improvements in the sensitivity of mass analyzers, speed of data processing, and data quality have been the primary focus of research across the proteomics field. Yet, hundreds, or even thousands, of samples must be analyzed to establish statistically significant and actionable information. This puts an enormous burden on proteomic sample preparation efforts to increase throughput and minimize variation despite protocols that involve many time-consuming, labor-intensive steps. Typical sample preparation protocols include over 20 liquid transfer events, reagent

Received: July 8, 2019

Published: August 22, 2019 
mixing, various centrifugation and incubation steps, and an extended tryptic digestion step.

Recently, several automated sample preparation protocols have been developed for blood plasma, urine, or other soluble protein samples. $^{6-10}$ However, protocols that incorporate automated cell lysis are much more difficult to implement because traditional cell lysis protocols such as sonication or shear stress via a French press are not easily applied in platebased formats compatible with liquid handling systems. Consequently, multiple efforts have been made to develop plate-based sample preparation protocols based on chemical lysis procedures such as filter-aided sample preparation $(\text { FASP })^{8,11,12}$ and chaotropes-like urea. ${ }^{13}$ These types of protocols, such as the Quick 96FASP ${ }^{8}$ method, require multiple buffer-exchange steps to remove detergents and chaotropes prior to tryptic digestion. This is a significant limitation for these protocols because molecular weight cut off (MWCO) filter plates compatible with liquid handling systems cannot be centrifuged as fast as the analogous spin columns that are used for the manual preparation protocols. ${ }^{14,15}$ Consequently, these protocols are time consuming and susceptible to variable sample loss. Efforts to circumvent the MWCO filter plate limitations by using polyvinylidene fluoride membranes have been shown to overcome the time limitation but produce missed cleavages and poor quantitative reproducibility. ${ }^{15}$ Similarly, tip-based peptide cleanup steps have been implemented in 96-well plate formats, ${ }^{16}$ however, at significant per-sample added expense.

In an effort to reduce variability and improve throughput, we developed an automated sample preparation workflow that consists of cell lysis, protein precipitation, protein resuspension, protein quantification, and normalization of protein concentration followed by standard bottom-up proteomic procedures of reducing and blocking cysteine residues and tryptic digestion. This comprehensive workflow can process four 96-well plates (384 samples) in parallel in under $6 \mathrm{~h}$ for subsequent analysis via liquid chromatography-mass spectrometry systems. We evaluated the effectiveness of workflow in a 96-well plate format against manual sample preparation protocols and demonstrated its effectiveness on Gram-negative bacteria (Escherichia coli, Pseudomonas putida) and fungi ( Saccharomyces cerevisiae, Rhodosporidium toruloides). We optimized protein extraction conditions and measured the variation of the workflow with high-throughput UHPLC-MRM mass spectrometry assays for this workflow.

\section{EXPERIMENTAL SECTION}

\section{Strains, Media, and Growth Conditions}

E. coli strain BW25113 (JPUB_001327), S. cerevisiae S288C (JPUB_013514), R. toruloides NP11 (JPUB_012600), and $P$. putida KT2440 (JPUB_012605) were cultured in house under the following conditions. For shake flask culturing at $200 \mathrm{rpm}$, E. coli and P. putida strains were grown for $24 \mathrm{~h}$ in Luria broth (LB) medium at 37 and $30^{\circ} \mathrm{C}$, respectively. S. cerevisiae and $R$. toruloides strains were grown overnight in YPD medium at 30 ${ }^{\circ} \mathrm{C}$. Cells were distributed to 96-well plate format and harvested by centrifugation. Cell pellets were frozen at -80 ${ }^{\circ} \mathrm{C}$ until further processing. For cultivation in 96-deep-well and BioLector plate formats, an overnight $E$. coli pre-culture was prepared in LB medium, and was inoculated to either 96-deep well plate, or a BioLector 48-well flat-bottom plate at 0.1 starting $\mathrm{OD}_{600}$ of a total $800 \mu \mathrm{L}$ culture volume in LB medium.
The 96-deep well plate was cultured for $24 \mathrm{~h}$ at $37^{\circ} \mathrm{C}$, shaking at $800 \mathrm{rpm}$. The BioLector plate experiment was run on a BioLector microbioreactor (m2p-labs, Hauppauge, NY) at 800 rpm at $37^{\circ} \mathrm{C}$ and ambient chamber pressure for $24 \mathrm{~h}$ with $85 \%$ humidity. For both experiments, cell biomass were harvested after cultivation by centrifugation $\left(3000 \mathrm{~g}\right.$ for $5 \mathrm{~min}$ at $4{ }^{\circ} \mathrm{C}$ ) and then stored at $-80{ }^{\circ} \mathrm{C}$ until prepared via the automated workflow.

\section{Manual Proteomic Sample Preparation}

Manual cell lysis and protein extraction was accomplished using a chloroform-methanol precipitation method as previously described. ${ }^{17}$ Briefly, for extracting proteins from samples in 96 well plates, the same chloroform-methanol cell lysis and protein extraction procedure is applied and the volume of reagents is reduced by a factor of 5. Vortexing is substituted by pipette mixing. Cell pellets were resuspended in $80 \mu \mathrm{L}$ of methanol and $20 \mu \mathrm{L}$ of chloroform and thoroughly mixed by pipetting. Water $(60 \mu \mathrm{L})$ was subsequently added to samples and mixed by pipetting. Phase separation was induced by centrifugation at maximum speed in a table centrifuge for $10 \mathrm{~min}$. The methanol and water layers were removed, and then $80 \mu \mathrm{L}$ of methanol was added to each well. The plate was centrifuged for another $10 \mathrm{~min}$ at maximum speed, then the supernatant chloroform and methanol layers were decanted. All resultant protein pellets were resuspended in $100 \mathrm{mM}$ ammonium bicarbonate (AMBIC) buffer supplemented with $20 \%$ methanol, and protein concentration was determined by the DC assay (BioRad, Hercules, CA). Prior to protein trypsin digestion at a concentration of $1 \mathrm{mg} / \mathrm{mL}$, protein reduction was accomplished using $5 \mathrm{mM}$ tris 2-(carboxyethyl)phosphine (TCEP) for $30 \mathrm{~min}$ at room temperature, and alkylation was performed with $10 \mathrm{mM}$ iodoacetamide (IAA) for $30 \mathrm{~min}$ at room temperature in the dark. Overnight digestion with trypsin was accomplished with a $1: 50(\mathrm{w} / \mathrm{w})$ trypsin/total protein ratio.

\section{Automated Proteomic Sample Preparation}

The automated sample preparation workflow was executed on a Biomek FXp system (Beckman-Coulter, Indianapolis, IN) with integrated SpectraMax Paradigm Multi-mode microplate reader (Molecular Devices), Sigma centrifuge (model 6-16K) (Sigma-Aldrich, St. Louis, MO), and a Cytomat incubator and plate hotel units (ThermoFisher Scientific, Waltham, MA). The workflow consists of 10 steps: cell biomass preparation and lysis, protein precipitation, remove metabolites, remove lipids, protein resuspension, protein quantification, protein normalization, and digestion with trypsin (Table S1). The cell biomass was prepared by resuspending cells in water, transferring the cells into a 96-well protein extraction plate, pelleting the cells by centrifugation for $10 \mathrm{~min}$, and subsequent removal of the water. The protein was extracted by transferring methanol and chloroform in a $4: 1(\mathrm{v} / \mathrm{v})$ ratio into the protein extraction plate with the cells with mixing to promote cell lysis. Next, water was added to the solution with mixing followed by centrifugation ( $5 \mathrm{~min}, 1000 \mathrm{~g}$ ) to promote phase separation. The top layer (methanol-water) was then discarded or filtered through a $0.45 \mu \mathrm{m}$ filter plate and stored for potential metabolite analysis at a later time. An additional aliquot of methanol was added to the protein extract followed by centrifugation ( $1 \mathrm{~min}, 100 \mathrm{~g}$ ) to pellet the protein at the bottom of the well. At this point, the remaining methanol and chloroform mixture was either discarded or filtered through a $0.45 \mu \mathrm{m}$ filter plate and stored for lipid analysis if so desired. 


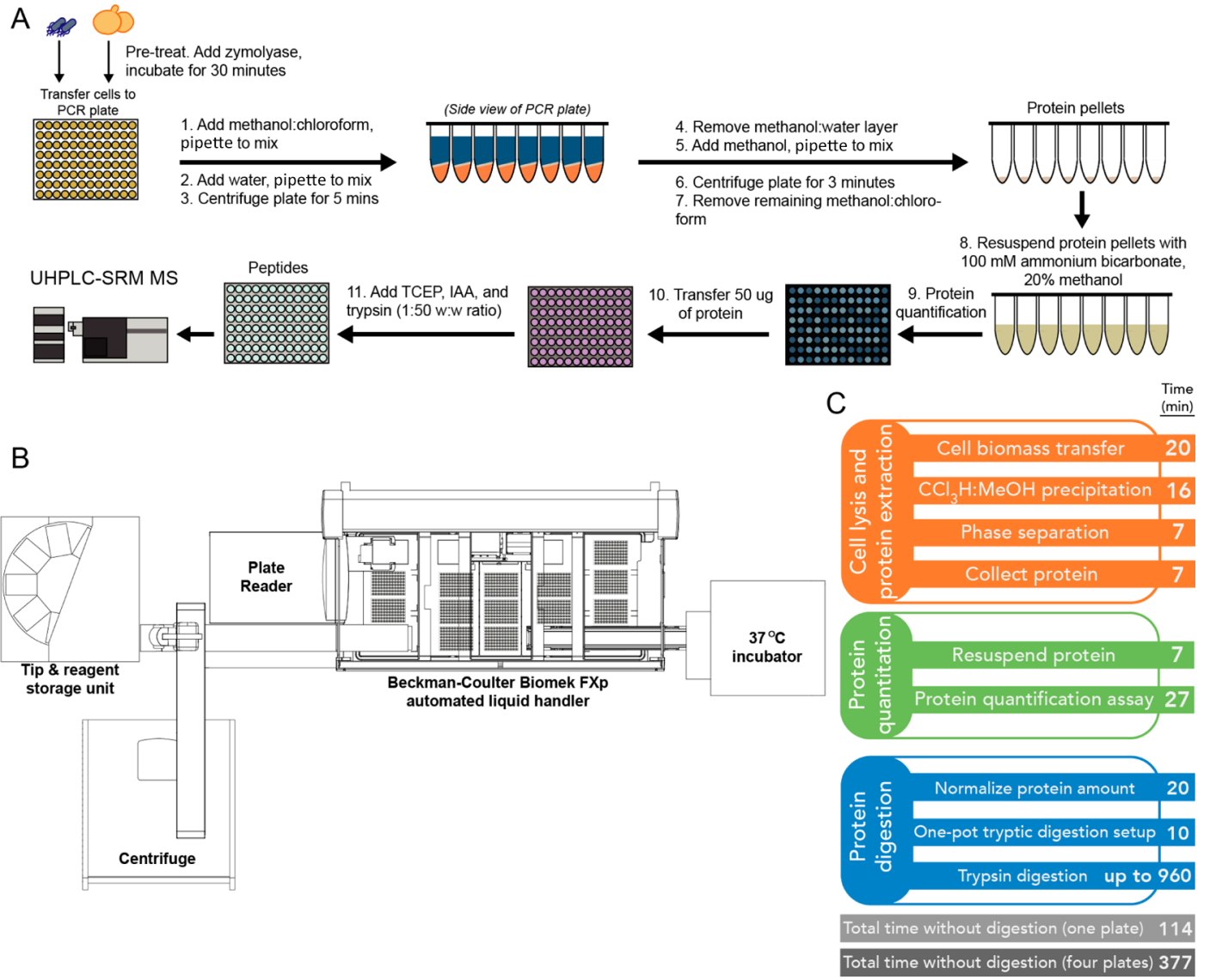

Figure 1. Automated sample preparation protocol. (A) Automated sample preparation steps; (B) automated liquid handling system layout; (C) timeline of the full workflow. Detailed times and reagents for each step can be found in Table S1.

The protein pellet was resuspended by addition of $100 \mathrm{mM}$ AMBIC supplemented with $20 \%$ methanol. Initial efforts to resuspend the precipitated protein resulted in poor protein quantification because of weaker aspiration with the automated liquid handler than what is possible via manual aspiration. To achieve better protein resuspension, we performed 80 cycles of pipetting mixing at the maximum allowable aspiration and dispensing speed. The protein concentration was measured using a Bio-Rad DC protein assay kit and a spectrophotometer operated in the automation mode. The concentration of each sample was communicated from the plate reader to the liquid handler software, and the volume necessary to transfer $50 \mu \mathrm{g}$ of protein was calculated and transferred to a new plate on the deck, the concentration was normalized to $1 \mu \mathrm{g} / \mu \mathrm{L}$ by adding $100 \mathrm{mM}$ AMBIC for subsequent trypsin digestion. A one-pot trypsin digestion mixture of TCEP, IAA, and trypsin was subsequently added into normalized protein solution to final concentrations of $5 \mathrm{mM}, 10 \mathrm{mM}$, and $1: 100(\mathrm{w} / \mathrm{w})$ ratio, respectively. Finally, the deck of the liquid handling system was cleaned up and all used tip boxes and solvents were removed. Once trypsin was added, digestion was carried out either by a programmed thermocycler (not integrated with the automation platform) or sealed and transported to the integrated Cytomat incubator at $37^{\circ} \mathrm{C}$ for $4 \mathrm{~h}$ or overnight.

\section{MRM Methods and UHPLC-MS Data Acquisition}

Targeted MRM methods were developed with the assistance of in-house spectral libraries of microbes. ${ }^{18}$ MRM selection criteria excluded peptides with Met/Cys residues, tryptic peptides followed by additional cut sites (KK/RR), and peptides with proline adjacent to $\mathrm{K} / \mathrm{R}$ cut sites. All possible doubly charged peptides were screened for $y$-series ions to establish the peptide identity and the most sensitive transitions. The MRM-targeted proteomic assays were performed on an Agilent 6460QQQ mass spectrometer system coupled with an Agilent 1290 UHPLC system (Agilent Technologies, Santa Clara, CA). Mobile phase A consisted of $0.1 \%$ FA (Thermo Scientific) in LC-MS grade water (Burdick \& Jackson, Muskegon, MI), and mobile phase B consisted of $0.1 \%$ FA in LC-MS grade acetonitrile (Burdick \& Jackson). Twenty microgram peptides were separated on an Ascentis Express Peptide C18 column [2.7 mm particle size, 160 Å pore size, 5 $\mathrm{cm}$ length $\times 2.1 \mathrm{~mm}$ inside diameter (ID), coupled to a $5 \mathrm{~mm}$ $\times 2.1 \mathrm{~mm}$ ID guard column with the same particle and pore size, operating at $60{ }^{\circ} \mathrm{C}$; Sigma-Aldrich, St. Louis, MO] operating at a flow rate of $0.4 \mathrm{~mL} / \mathrm{min}$ via gradients depending on the organism of our investigations. To comprehensively evaluate the variance of automated sample preparation platform for the microorganism we investigated, highly multiplexed assays that target hundreds of peptides were developed in either a single, scheduled $25 \mathrm{~min}$ gradient UHPLC-MRM-MS analysis for E. coli or a single, scheduled 30 min gradient UHPLC-MRM-MS analysis for the other three microorganisms. For the $25 \mathrm{~min}$ LC run, peptides were loaded to the column equilibrated with $5 \% \mathrm{~B}$ and hold for 0.6 min, followed by a linear gradient elution to $35 \%$ B over 20.4 min. The column was washed at $80 \% \mathrm{~B}$ for $2 \mathrm{~min}$ and then equilibrated to $5 \% \mathrm{~B}$ for $1.5 \mathrm{~min}$ before loading the next sample. For the $30 \mathrm{~min}$ LC run, peptides were loaded to the 
column equilibrated with $4 \% \mathrm{~B}$ and hold for $1 \mathrm{~min}$, followed by a linear gradient elution to $40 \% \mathrm{~B}$ over $20 \mathrm{~min}$. The column was washed at $90 \%$ B for $2 \mathrm{~min}$, and then equilibrated to $4 \%$ B for $4 \mathrm{~min}$ before loading the next sample. The eluted peptides were ionized via an Agilent Jet Stream ESI source operating in the positive ion mode with the following source parameters: gas temperature $=250{ }^{\circ} \mathrm{C}$, gas flow $=13 \mathrm{~L} / \mathrm{min}$, nebulizer pressure $=35 \mathrm{psi}$, sheath gas temperature $=250^{\circ} \mathrm{C}$, sheath gas flow $=11 \mathrm{~L} / \mathrm{min}$, capillary voltage $=3500 \mathrm{~V}$, nozzle voltage $=0$ $\mathrm{V}$. The transitions are acquired in the dynamic MRM mode with scanning retention time window of $0.6 \mathrm{~min}$ and a total cycle time of $1 \mathrm{~s}$. All MRM transitions $\mathrm{m} / z$ values are included in Tables S2-S5. The data were acquired using Agilent MassHunter version B.08.02. MRM methods were generated, and all acquired data were analyzed by Skyline software version 4.20 (MacCoss Lab Software) and are available along with additional method details on Panoramaweb (https:// panoramaweb.org/automated-sample-prep.url). The MRM peptide quantitation results were combined into total peak area per protein. The data were analyzed by using a custom python script to quantify and visualize error. For each dataset, mean, variance, and coefficient of variation $(\mathrm{CV})$ were computed for replicates. The data were visualized with violin plots for each condition showing the distribution of the coefficients of variation across all proteins and peptides in the data set. Scatter plots were created that show the relationship between mean and CV for all proteins and peptides in the data set. The primary dataset was visualized by using Plotly for violin and box-and-whisker plots.

\section{RESULTS AND DISCUSSION}

\section{Automated Bottom-Up Proteomic Protocol for Microbes}

As the number of proteomic samples increases, traditional manual proteomic sample preparation procedures become a bottleneck in terms of resources and data quality. Thus, efforts to automate liquid transfer steps of cell lysis, protein extraction, and digestion are attractive to both minimize variation and improve resource utilization. While several automated sample preparation protocols have been developed previously, they utilize detergents or chaotropes that must be removed prior to LC-MS analysis. Consequently, one goal of this work was to minimize the steps needed to remove compounds that are used for cell lysis but are detrimental to LC-MS analysis. The chloroform-methanol (C/M) protocol ${ }^{19}$ effectively lyses and precipitates protein from a wide variety of microbial organisms, and it is routinely used for manual sample preparation of proteomic samples from Gram-negative bacteria. ${ }^{4,17,20}$ For this automated workflow, we established the utility and reproducibility of the $\mathrm{C} / \mathrm{M}$ protocol for a plate-based proteomic sample preparation workflow (Figure 1A) applied to both Gram-negative bacteria and, by pretreatment with zymolyase to digest the cell wall glucan, fungal species such as $S$. cerevisiae and $R$. toruloides. One of the primary challenges of applying the $\mathrm{C} / \mathrm{M}$ protocol in a plate-based format is isolation of the protein that precipitates at the interface between the chloroform and methanol layers. However, by removing the upper methanol-water layer with the automated liquid handling system (Figure 1B), followed by the addition of a second aliquot of methanol, the protein pellet settles to the bottom of the plate. This allowed facile removal of the methanol-chloroform mixture via liquid handling systems, thereby simplifying subsequent processing steps. Here, the precipitated protein was resuspended and all 96 wells were quantified by using a Lowry-based protein quantification method via a plate reader integrated into the liquid handler system (Figure 1A). After the concentration of protein in each well was measured, $50 \mu \mathrm{g}$ of protein was transferred automatically to a new 96-well plate for reduction with TCEP, blocking of cysteine moieties by using IAA, and digestion with trypsin. Sample preparation of cells subjected to the $\mathrm{C} / \mathrm{M}$ protocol described above take $2 \mathrm{~h}$ from the beginning of the lysis step to the addition of trypsin (Figure 1C) for one 96-well plate. The entire workflow takes $377 \mathrm{~min}(6.3 \mathrm{~h})$ to prepare 384 samples (four 96-well plates) from cell biomass to the beginning of tryptic digestion.

\section{Optimization of the Automated "Cells-To-Peptides"} Protein Extraction Process

To evaluate the effectiveness of $\mathrm{C} / \mathrm{M}$ precipitation on variable amounts of cellular biomass in plate format, a single $E$. coli culture was grown overnight at $37^{\circ} \mathrm{C}$ in LB media, diluted to different cell densities, and distributed across a 96-well PCR plate. Estimates based on optical density can be highly variable while accurate cell count procedures are low throughput, consequently, for this comparison, cell biomass corresponding to $0.5,1.0,2.0$, and $3.0 \mathrm{OD}^{*} \mathrm{~mL}\left(1 \mathrm{OD}=\sim 5 \times 10^{8} \mathrm{E}\right.$. coli cells) of culture were aliquoted into 96-well deep plates $(n=$ 96 for each amount of cells). The cells were centrifuged and the supernatant was decanted by inverting the plate, after which the cells were subjected to automated $\mathrm{C} / \mathrm{M}$ cell lysis, resolubilization with a mixture of $100 \mathrm{mM}$ AMBIC/methanol $(80 \% / 20 \%)$, followed by protein quantification. The amount of protein extracted followed a near-linear increase from the different amounts of cells with the median protein amounts of 18 , 56, and $112 \mu \mathrm{g} /$ well from $0.5,1.0$, and $2.0 \mathrm{OD}^{*} \mathrm{~mL}$, respectively (Figure $2 \mathrm{~A}$ ). The protein recovery efficiency of the automated protocol was assessed by comparison to the total extractable protein by manual sample preparation (Figure 2B) of $0.5,1.0$, and $2.0 \mathrm{OD}^{*} \mathrm{~mL}$ of cells prepared in the plate

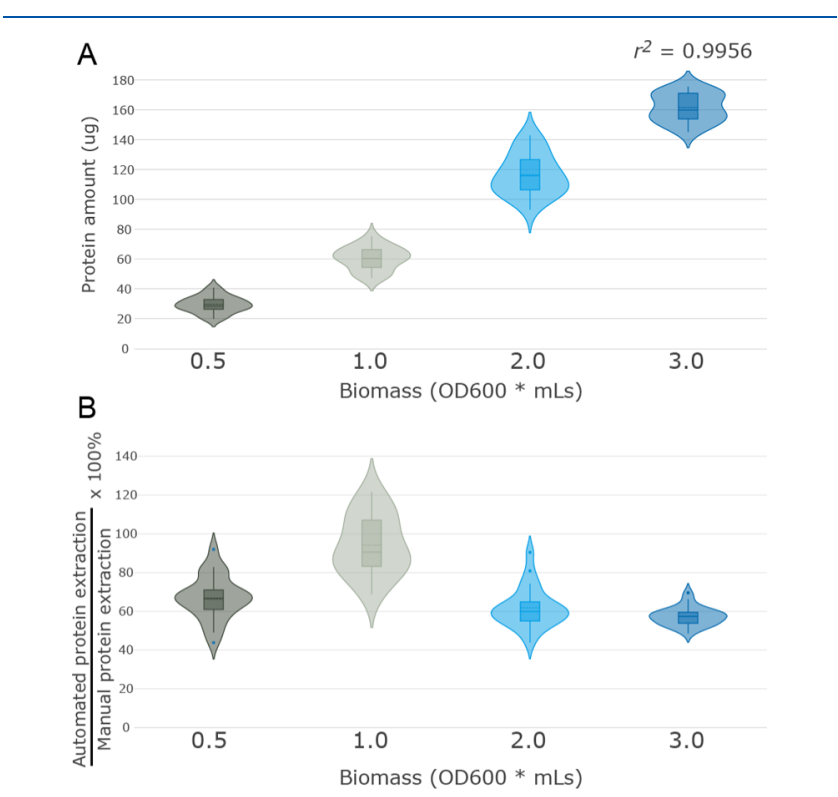

Figure 2. Comparison of automated sample preparation workflow protein extraction (A) and protein extraction efficiency (automated/ manual process) (B) from different amounts of E. coli cell biomass. The cell biomass amounts and their average protein amount yields fit a simple linear regression model of $r^{2}=0.9956$. 


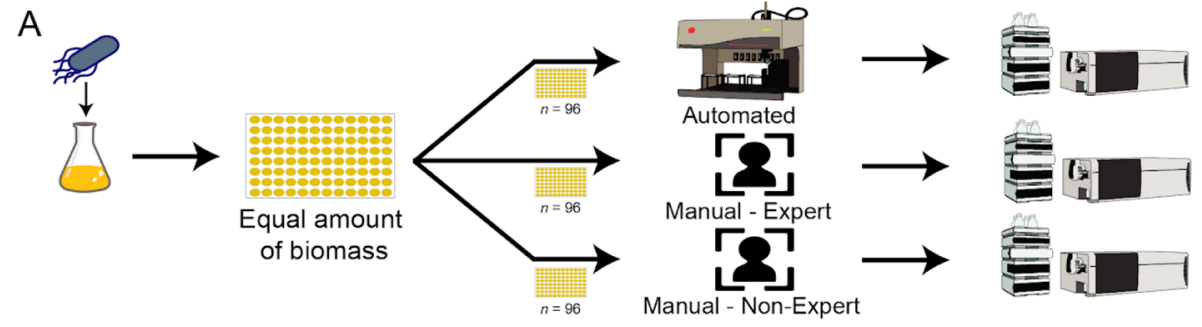

B
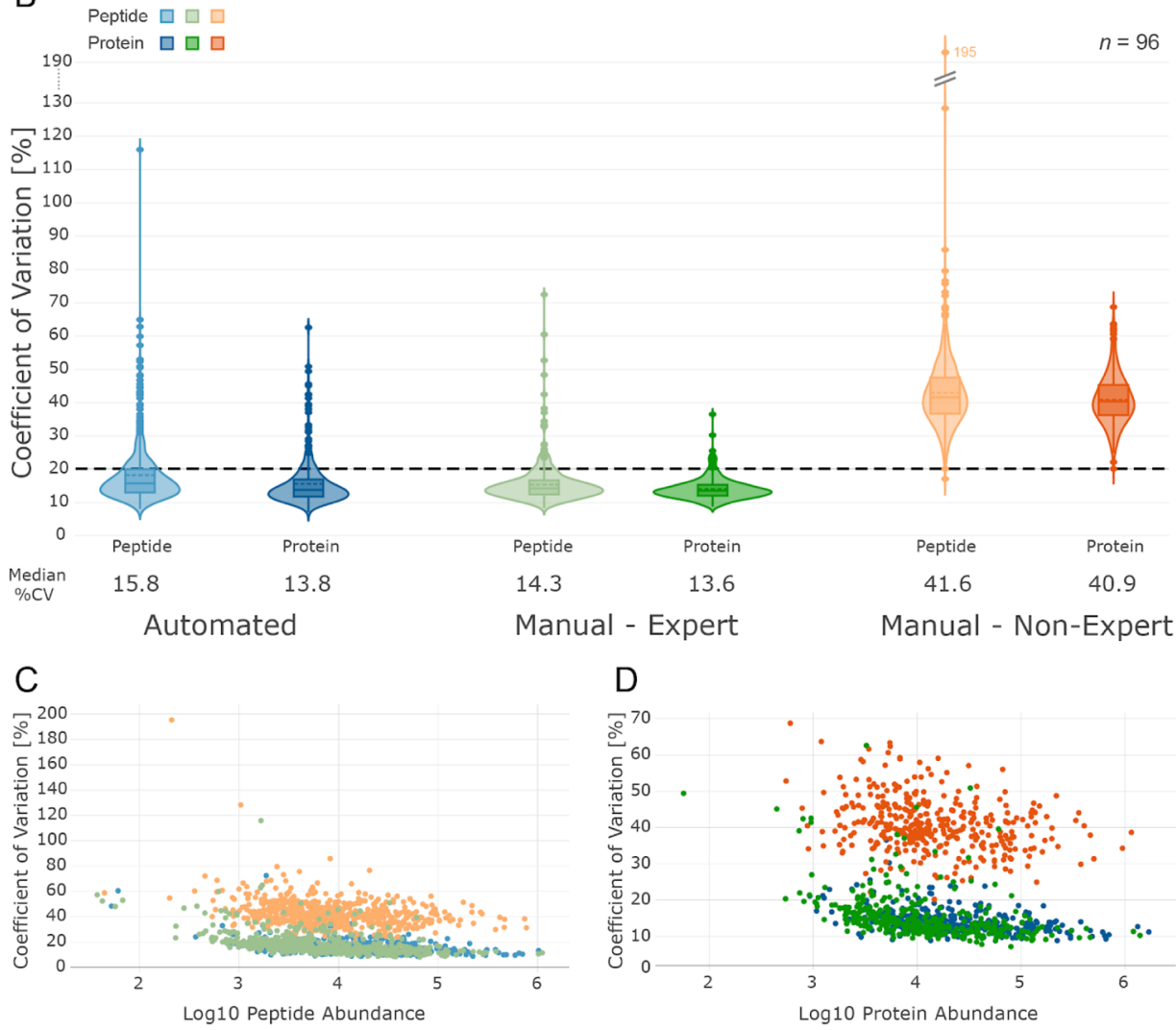

Figure 3. Reproducibility of the automated and manual sample preparation workflows as measured by UHPLC-MRM-MS data acquisition. (A) Experiment design and (B) violin and scatter plots of the percent CV (\% CV) for 600 peptides (C) from 367 E. coli proteins (D). The violin plots illustrate the kernel density estimation of the $\mathrm{CV}$ of the automated, manual-expert, and manual-nonexpert workflows. Inside each violin plot is a box plot summarizing ranges (IQR, whiskers) and individual medians (solid lines), averages (dashed lines) and the black dotted line indicates the $20 \%$ $\mathrm{CV}$ value. The scatter plots plot the \% CV for each peptide and protein $(y$-axis) vs the log 10 of the peptide intensity ( $x$-axis) (E). The total amount of protein loaded on the UHPLC-MS system were normalized during sample preparation, but the peptide counts were not normalized postacquisition.

format with multi-channel pipettes. Automated cell lysis and protein precipitation experiments $(n=24)$ indicated that more protein was lost for 0.5 and $2.0 \mathrm{OD}^{*} \mathrm{~mL}$ and above of cells likely because of poor pellet formation or because of protein remaining in the methanol-water solution and removal via pipetting. Given the plate format and the volumes of the reagents selected, the most efficient process was observed for $1.0 \mathrm{OD}^{*} \mathrm{~mL}$ of cells. However, to ensure a sufficient amount of peptide digest for data acquisition and minimize liquid transfer variation, we selected $2.0 \mathrm{OD}^{*} \mathrm{~mL}$ of $E$. coli cell biomass for subsequent experiments. Likewise, resolubilization of $R$. toruloides and P. putida proteins showed similar amounts and variability to $E$. coli, indicating that this protocol is applicable to a wide variety of microbial organisms. This shows that performing the $\mathrm{C} / \mathrm{M}$ protocol and resuspending the protein under these conditions yields highly reproducible amounts of protein that is sufficient for common nano-flow and standard- flow LC-MS methods. Optimization of the workflow for smaller or larger initial biomass amounts could be achieved by increasing centrifugation force and time or by adjusting the volume of methanol and chloroform used for the protein extraction step as well as adjusting the height of the pipetting step to remove the methanol-water layer.

Reproducibility of the Automated Workflow Measured by Targeted Proteomic Assays

Once the optimized automated $\mathrm{C} / \mathrm{M}$ method was established, we assessed the reproducibility of the automated workflow against expert and non-expert manual sample preparation protocols via targeted proteomic assays. For comparison, the same amount $E$. coli biomass $\left(2 \mathrm{OD}^{*} \mathrm{~mL}\right)$ was distributed across a plate (96 replicate samples) and prepared either via the automated workflow or manually by using multi-channel pipettes by an expert in proteomics sample preparation that had experience preparing over 2000 samples with the $\mathrm{C} / \mathrm{M}$ 
protocol and by a nonexpert who had experience preparing over 100 samples with the $\mathrm{C} / \mathrm{M}$ protocol (Figure 3A). Fifty micrograms of protein from the automated or manual $\mathrm{C} / \mathrm{M}$ extraction protocols were digested at a final concentration of 1 $\mu \mathrm{g} / \mu \mathrm{L}$, and $20 \mu \mathrm{L}$ was loaded onto a reverse-phase $\mathrm{C} 18$ chromatography column connected to an UHPLC-QQQ system operating in the dynamic MRM mode. The MRM method (available at: https://panoramaweb.org/automatedsample-prep.url) targeted 600 peptides from $367 \mathrm{E}$. coli proteins known to be expressed under these culture conditions. Among the three methods, we observed that the automated workflow produced similar variance for peptide measurements to that of the expert, whereas the highest variance data was produced by the nonexpert. The median $\mathrm{CV}$ for the automated workflow was 15.8 and $13.8 \%$ for peptide and protein measurements, respectively (Figure 3B). Importantly, $75 \%$ of the total peptide CVs were below 20\%. As expected, the majority of peptides and proteins with high variance had the lowest intensity, and an overall trend of decreasing variance with increasing signal intensity was observed (Figure 3C,D), suggesting that low signal intensity was the main factor in high technical variability. Even though the automated sample preparation workflow showed slightly higher variance, overall it achieved similar performance as experts in the sample preparation field. The inter-day reproducibility of the automated sample preparation platform was evaluated by assaying plate samples that were prepared on three separate days. The median CVs of 600 peptides from these separate assessments ranged from 15.8 to $19.2 \%$, suggesting that the use of automation for sample processing provides good day-to-day consistency (Figure S1).

Automated protein extraction from industrially favored bacteria $P$. putida demonstrated another successful application of the automated platform on Gram-negative bacteria. The extension of its application on other organisms, such as $S$. cerevisiae and $R$. toruloides in our tests, requires additional procedure of weakening their cell wall structure and more starting biomass. Similarly, we distributed cell biomass from single cultures of these microorganisms, and investigated the variances presented in the peptide samples that were prepared through the automated workflow. Highly multiplexed MRM assays were developed for targeting 340, 305, and 401 peptides in $P$. putida, $S$. cerevisiae, and $R$ toruloides, respectively. Our results showed similar variance distributions on these microorganisms in comparison to what we have observed in the tests of E. coli cell culture (Figure 4). As observed for E. coli, low peptide signal intensity was the main factor in high technical variability. The peptide median CV achieved at 11.6, 14.7, and $15.8 \%$ for P. putida, S. cerevisiae, and R. toruloides, respectively. The similar $\mathrm{CV}$ distributions observed for these organisms suggest that the automated sample preparation workflow is applicable for a wide variety of applications.

\section{Identification of Sources of Variance in the Automated Sample Preparation Workflow}

To identify the sources of variance across the workflow, we isolated several steps in the process and assessed the error of each one. As shown in Figure 2 above, we observed protein extraction variations from distributed biomass samples in plates, however, this variation should have no contribution to the overall variance because the amount of protein that was digested for LC-MS acquisition was normalized after determining the concentration. Consequently, the variance is

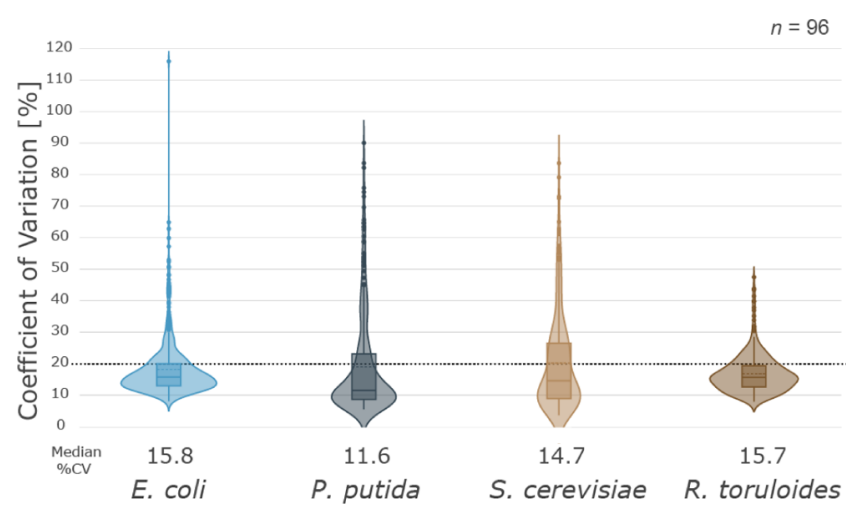

Figure 4. Reproducibility of the automated sample preparation workflow as measured by UHPLC-MRM-MS data acquisition for four different organisms. (A) Violin plots of the \% CV for 600, 340, 305, 401 peptides from E. coli, $P$. putida, S. cerevisiae, $R$. toruloides proteins, respectively $(n=96)$. The total amount of protein loaded on the UHPLC-MS system was normalized but the peptide counts were not normalized post-acquisition.

likely due to the protein quantification, normalization, and the tryptic digestion steps. We tested the variability of the protein quantification step by distributing two concentrations (1.9 and $4.8 \mu \mathrm{g} / \mu \mathrm{L}$ ) that were determined manually via the DC protein quantification assay of a whole-cell lysate protein mixture produced from a single culture, across two 96-well plates and subjected them to the automated protein quantitation process. The same protein samples were quantified manually by using the same Bio-Rad DC assay ( $n=36$ each). We observed CVs of 2.0 and $3.5 \%$ with the manual assay and CVs of 5 and $7 \%$ with the automated protocol for the 1.9 and $4.8 \mu \mathrm{g} / \mu \mathrm{L}$ samples, respectively. When performing the protein resuspension step manually, it is easy to meticulously resuspend the protein with extra pipetting force and time, thus, leading to more accurate protein quantification and normalization steps. This factor likely contributes to the small CV distribution difference observed between the samples prepared by human expert and the automated workflow above. Future workflow optimization efforts will focus on improving this step in the automated workflow.

To investigate the possible sources of the observed variation in the tryptic digestion step, we distributed $50 \mu \mathrm{g}$ of protein that was extracted from a single $E$. coli cell culture, across a 96well plate and initiated the automated workflow from the trypsin digestion step (Figure 5A). By using the same MRM method that targeted 600 peptides to assess the total variance from tryptic digest to LC-MS acquisition procedures, the median peptide $\mathrm{CV}$ for the trypsin digestion components was $8.7 \%$ (Figure $5 \mathrm{~B}$ ) with over $89 \%$ of peptide CV under $20 \%$. This error along with approximately $7 \%$ of the error observed in the complete automated workflow coming from the protein quantification and normalization steps accounts for essentially all of the variability ( $16.1 \%$ for peptides) in the process.

A common practice to improve the data acquisition accuracy and reduce variance is to introduce internal standards into the samples at different steps of the proteomic sample preparation process. $^{21}$ These standards could be stable-isotope-labeled proteins, stable-isotope-labeled synthetic peptides, or an endogenous protein that is considered to be constant in the tested samples. We tested the normalization effect to internal standards for the automated workflow at two points in the process; First, by spiking a constant amount of peptides from a 
A

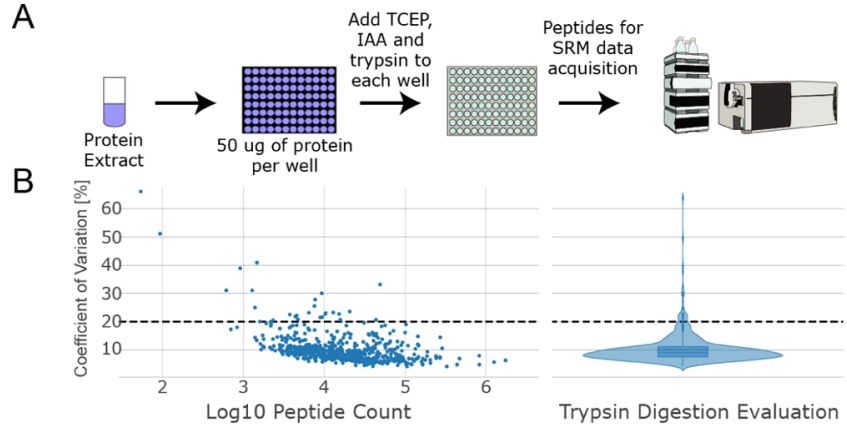

C

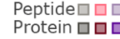

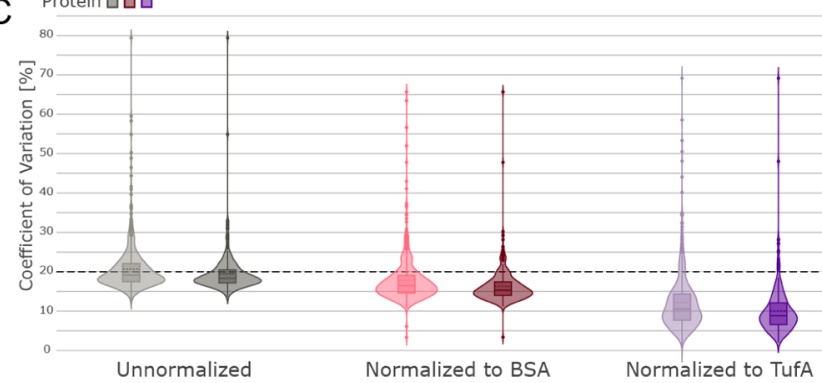

Figure 5. Identification of the sources of variation in the automated sample preparation process. (A) Tryptic digest experimental design and (B) scatter and violin plots of the \% CV for 600 peptides from 367 E. coli proteins $(n=96)$. (C) Comparison of unnormalized, bovine serum albumin (BSA)-normalized, and TufA-normalized \% CVs from the automated sample preparation workflow for 600 peptides from $367 \mathrm{E}$. coli proteins $(n=96)$. The total amount of protein loaded on the UHPLC-MS system was normalized and the peptide counts were normalized postacquisition to internal standards as indicated.

heterologous protein, tryptically digested peptides of BSA, into each sample at the end of the workflow prior to data acquisition to determine the contribution of the LC-MS analysis to the observed variation; second, by normalizing the data to an endogenous E. coli protein, elongation factor Tu-1 (TufA), to account for variance across the entire automated workflow. The peak areas of 600 peptides, as measured from 96 replicate samples prepared by the automated workflow, ranged from $10^{3}$ to $>10^{6}$ counts with median CVs of $19 \%$ (59\% below $20 \% \mathrm{CV}), 16 \%(80 \%)$, and $11 \%(89 \%)$ for unnormalized, BSA-normalized, and TufA-normalized, respectively (Figure 5C). This improvement of data quality could be observed in all the tests we have presented above, suggesting that introducing internal standards from the beginning of the automation process greatly improves data acquisition accuracy and reduce technical variance. This workflow is well-suited for Qualis-SIS $^{22}$ or single-point external reference material ${ }^{23}$ data acquisition methods that have been developed for multiplexed, quantitative proteomic assays. Furthermore, the precision $(<20 \% \mathrm{CV})$ of this workflow is suitable for targeted peptide measurements across exploratory studies (tier 3 ) and, with appropriate isotopically-labeled internal standards, for clinical bioanalysis/diagnostics (tier 1) and quantitative research-use (tier 2) studies as proposed by Carr et al. ${ }^{24}$

Reproducibility of Protein Levels from Cells Cultured in Different Formats

Cultivation in multi-well plate formats is a useful way to facilitate large experimental designs and increase sample throughput. While growth has been shown to be reproducible in various plate-based culture conditions, the variance in the proteomes of cells from different formats has not been comprehensively characterized. We tested whether culturing cells in low volume growth format, such as 96-deep well plate or BioLector ${ }^{25,26}$ flower plate, would increase the variance among replicates in comparison to culturing in $5 \mathrm{~mL}$ test tube culture conditions. To assess this question, we grew E. coli BW25113 cells in LB medium for $24 \mathrm{~h}$ in three culture platforms and prepared peptides from them with the automated workflow (Figure 6A). The CVs of 357 E. coli proteins in 18 biological replicates after normalization to TufA showed overall low technical variance but slightly different at three cultivation platforms (Figure 6B). The test tube platform achieved the best median $\mathrm{CV}$ at $5.4 \%$, followed by 96 -deep well plate at $5.5 \%$, and BioLector plate at $11.4 \%$ sequentially. Notably, over $95 \%$ proteins measured in the test tube and 96deep well plate platforms achieved CV below $20 \%$. The three cultivation platforms were well separated in principalcomponent analysis (PCA) based on their peptide quantity differences (Figure 6C), suggesting that there are differences between the proteomes of the three cultivation platforms. However, there was a relatively high correlation between them with no correlation dropping below 0.85 (Figure 6D) across cultivation condition and extremely high $(>99 \%)$ correlation within replicates of a given cultivation type.

The correlation analysis showed that the BioLector samples agree slightly more with culture tubes than does the 96-well plate format. The lower correlation may result from physiological differences during biological growth or microcultivation condition differences in the incubator. ${ }^{27}$ Pairwise comparison between plate formats and the test tube identified 127 genes as differentially expressed by more than 2 -fold with statistical significance ( $p$-value $=0.05$; Table S2). Interestingly, the majority of these genes (121) are down-regulated and are mostly identified in BioLector plate samples. Functional enrichment analysis (DAVID ${ }^{28}$ version 6.8) of these downregulated genes against the background of 367 measured proteins indicated that proteins involved in "protein synthesis and refolding" were highly enriched, including FKBP-type 22 $\mathrm{kDa}$ peptidyl-prolyl cis-trans isomerase (FklB), FKBP-type peptidyl-prolyl cis-trans isomerases (FkpA, SlyD), and trigger factor (Tig) as the highest scored enriched group. Additionally, the proteins from central carbon metabolism, particularly pentose phosphate pathway genes, including glucokinase (Glk), transketolase 1 (TktA), transketolase (TktB), transaldolase A (TalA), transaldolase B (TalB), fructose-bisphosphate aldolase class 1 (FbaB), and ATP-dependent 6phosphofructokinase isozyme 2 ( $\mathrm{PfkB}$ ) were enriched. Overall, our data suggest that the high-throughput cultivation platforms contribute negligible variance to proteome measurements, thus are as suitable to conduct experiments as using the traditional test tube.

\section{CONCLUSIONS}

Here, we present an automated proteomics workflow for cell lysis, protein precipitation, robust tryptic digestion (cells-topeptides), and data acquisition by LC-MRM mass spectrometry. Cell lysis and protein precipitation by using the chloroform-methanol protocol is amenable to parallel processing via automated liquid handling systems for a variety of cell types and efficiently isolates proteins from cellular lipids and metabolites. We demonstrated that the workflow is effective for quantitative proteomic studies of Gram-negative bacteria (E. coli, P. putida) and fungi such as S. cerevisiae and R. 


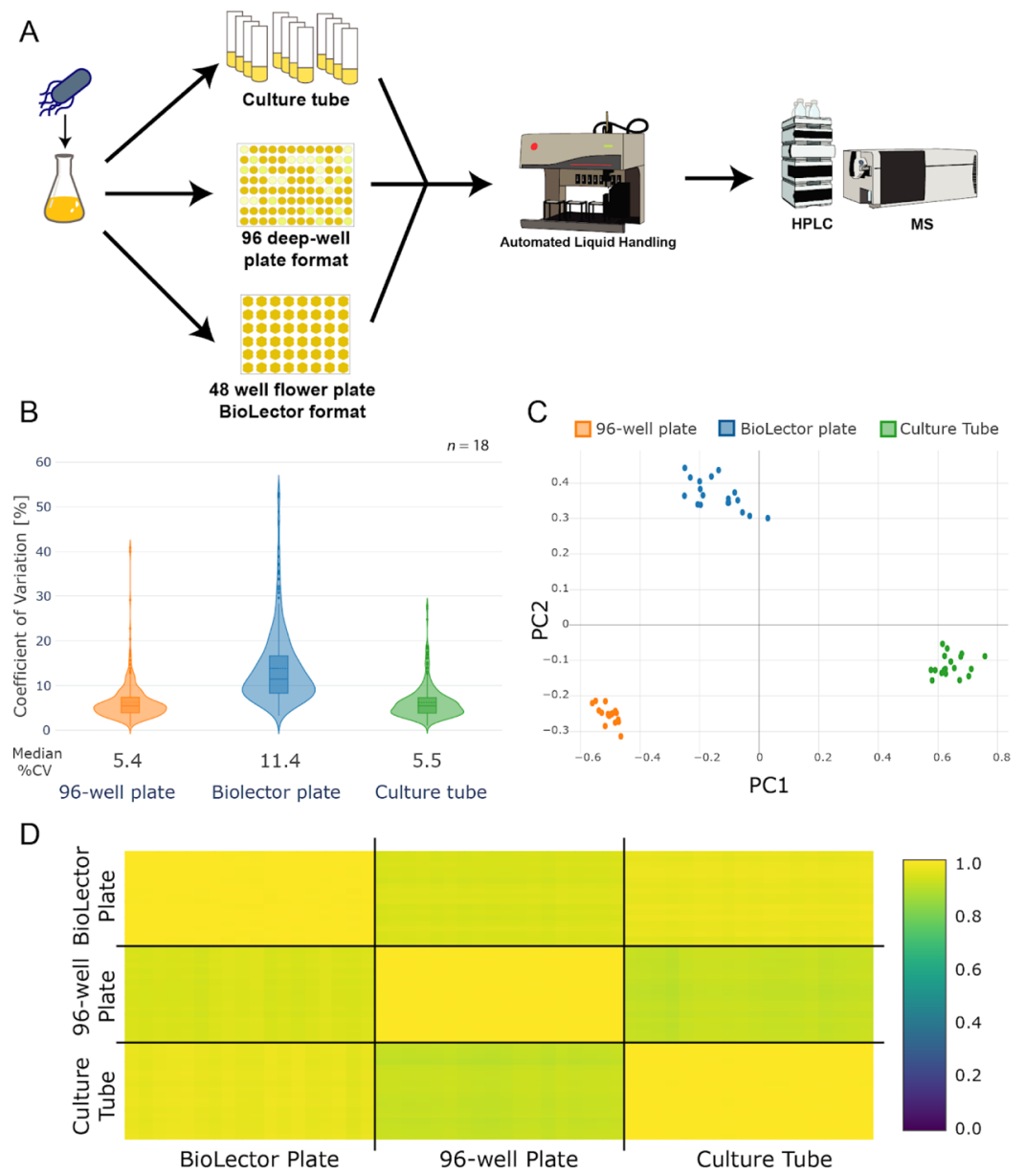

Figure 6. Variation analysis of culture condition samples (A) experiment design and (B) violin plots of the \% CV for 367 E. coli proteins ( $n=18$ ). (C) PCA demonstrates that protein expression for replicates in the same culture condition cluster tightly together. (D) A pairwise correlation analysis on replicates was performed to further determine how replicates relate to one another within and between formats. In all cases, the determined correlation coefficient was above 0.85 and showed good agreement between formats. The total amount of protein loaded on the UHPLC-MS system was normalized to $20 \mu \mathrm{g}$ and the peptide counts were normalized to TufA abundance post-acquisition.

toruloides in the plate format. The automated workflow was tested by preparing 96 E. coli samples and quantifying over 600 peptides that resulted in median CVs of $15.8 \%$. Further testing identified the protein resuspension and quantification steps as the biggest contributors to the observed variability. Importantly, the consistent performance of the automation platform would alleviate manual labor, risk of repetitive stress injuries, and the overall stress level of researchers that come from preparing thousands of proteomic samples.

The time required for the workflow can be optimized for specific experimental needs or resource availability. By using data acquisition methods of $10 \mathrm{~min}$ total duration, ${ }^{18}$ the workflow supports a throughput of 144 samples per day per instrument. While we applied this workflow to targeted proteomic methods, it is also useful for comparative shotgun proteomic experiments. This automated workflow can be modified to include recovery of the culture supernatant prior to $\mathrm{C} / \mathrm{M}$-mediated cell lysis permits analysis of secretome samples for protein production applications or integrated into fully automated cell culturing, sampling, and sample preparation experiments. Even though this workflow utilizes a highly specialized liquid handling system, the basic pipetting steps can be implemented on many off-the-shelf automation platforms while the centrifugation and protein quantification steps can be done manually. Future work will include extending the workflow to Gram-positive bacteria and mammalian cells, reducing variation in the protein quantification step and optimizing the metabolomic and lipidomic extractions so they can be paired with proteomics to provide a system-level view of the microbe. Overall, this automated sample preparation workflow provides robust, reproducible proteomic samples for high-throughput applications.

\section{ASSOCIATED CONTENT}

\section{Supporting Information}

The Supporting Information is available free of charge on the ACS Publications website at DOI: 10.1021/acs.jproteome.9b00455.

Violin plots of the inter-day variability of the automated workflow. Detailed time points and reagents for each step of the automated workflow. MRM transition parameters for E. coli. MRM transition parameters for P. putida. MRM transition parameters for $S$. cerevisiae. MRM transition parameters for $R$. toruloides. Upregulated and down-regulated proteins in cultivation platforms (PDF)

Six supplemental tables (XLSX) 


\section{AUTHOR INFORMATION}

\section{Corresponding Author \\ *E-mail: cjpetzold@lbl.gov. \\ ORCID $\odot$}

Jay D. Keasling: 0000-0003-4170-6088

Héctor García Martín: 0000-0002-4556-9685

Nathan J. Hillson: 0000-0002-9169-3978

Christopher J. Petzold: 0000-0002-8270-5228

\section{Present Addresses}

${ }^{\ddagger}$ Labcyte Inc., San Jose, CA, USA.

${ }^{\S}$ Calico Biosciences, South San Francisco, CA, USA.

\section{Author Contributions}

Conceptualization, Y.C. and C.J.P.; investigation, Y.C., J.M.G., L.J.G.C., T.L.O., H.M.T.., J.M.B.-H., and J.W.G.; formal analysis, Z.C. and Y.C.; visualization, Y.C., Z.C., and C.J.P.; supervision, J.D.K., H.G.M., N.J.H., and P.D.A.; writingoriginal draft, Y.C. and C.J.P; writing-review and editing, all the authors. All the authors have given approval to the final version of the manuscript.

Notes

The authors declare the following competing financial interest(s): J.D.K. has financial interests in Amyris, Lygos, Constructive Biology, Demetrix, Napigen and Maple Bio. All generated skyline files are available in the Panorama Public repository at this link: https://panoramaweb.org/automatedsample-prep.url and data are available via ProteomeXchange with identifier PXD014182.

\section{ACKNOWLEDGMENTS}

The authors thank Mark Forrer, Mark Kulawik, and William Morrell for help with informatics and data sharing. The proofof-concept work and resources were part of the Joint BioEnergy Institute (JBEI; http://www.jbei.org) and extension of the procedure for $R$. toruloides and identification of the sources of error were part of the Agile BioFoundry (ABF; http://agilebiofoundry.org) supported through contract DEAC02-05CH11231 between Lawrence Berkeley National Laboratory and the U. S. Department of Energy. The United States Government retains and the publisher, by accepting the article for publication, acknowledges that the United States Government retains a non-exclusive, paid-up, irrevocable, worldwide license to publish or reproduce the published form of this manuscript, or allow others to do so, for United States Government purposes. The views and opinions of the authors expressed herein do not necessarily state or reflect those of the United States Government or any agency thereof. Neither the United States Government nor any agency thereof, nor any of their employees, makes any warranty, expressed or implied, or assumes any legal liability or responsibility for the accuracy, completeness, or usefulness of any information, apparatus, product, or process disclosed, or represents that its use would not infringe privately owned rights. H.G.M. was also supported by the Basque Government through the BERC 2018-2021 program and by the Spanish Ministry of Economy and Competitiveness MINECO: BCAM Severo Ochoa excellence accreditation SEV-2017-0718.

\section{REFERENCES}

(1) Zhu, W.; Smith, J. W.; Huang, C.-M. Mass spectrometry-based label-free quantitative proteomics. J. Biomed. Biotechnol. 2010, 2010, 840518.

(2) Shi, T.; Fillmore, T. L.; Sun, X.; Zhao, R.; Schepmoes, A. A.; Hossain, M.; Xie, F.; Wu, S.; Kim, J.-S.; Jones, N.; et al. Antibody-free, targeted mass-spectrometric approach for quantification of proteins at low picogram per milliliter levels in human plasma/serum. Proc. Natl. Acad. Sci. U.S.A. 2012, 109, 15395-15400.

(3) Cominetti, O.; Núñez Galindo, A.; Corthésy, J.; Oller Moreno, S.; Irincheeva, I.; Valsesia, A.; Astrup, A.; Saris, W. H. M.; Hager, J.; Kussmann, M.; et al. Proteomic Biomarker Discovery in 1000 Human Plasma Samples with Mass Spectrometry. J. Proteome Res. 2016, 15, 389-399.

(4) Brunk, E.; George, K. W.; Alonso-Gutierrez, J.; Thompson, M.; Baidoo, E.; Wang, G.; Petzold, C. J.; McCloskey, D.; Monk, J.; Yang, L.; et al. Characterizing Strain Variation in Engineered E. coli Using a Multi-Omics-Based Workflow. Cell Syst. 2016, 2, 335-346.

(5) Aebersold, R.; Mann, M. Mass-spectrometric exploration of proteome structure and function. Nature 2016, 537, 347-355.

(6) Fu, Q.; Kowalski, M. P.; Mastali, M.; Parker, S. J.; Sobhani, K.; van den Broek, I.; Hunter, C. L.; Van Eyk, J. E. Highly reproducible automated proteomics sample preparation workflow for quantitative mass spectrometry. J. Proteome Res. 2018, 17, 420-428.

(7) Switzar, L.; van Angeren, J.; Pinkse, M.; Kool, J.; Niessen, W. M. A high-throughput sample preparation method for cellular proteomics using 96-well filter plates. Proteomics 2013, 13, 2980-2983.

(8) Yu, Y.; Bekele, S.; Pieper, R. Quick 96FASP for high throughput quantitative proteome analysis. J. Proteomics 2017, 166, 1-7.

(9) Dayon, L.; Núñez Galindo, A.; Corthésy, J.; Cominetti, O.; Kussmann, M. Comprehensive and Scalable Highly Automated MSBased Proteomic Workflow for Clinical Biomarker Discovery in Human Plasma. J. Proteome Res. 2014, 13, 3837-3845.

(10) Lee, J.; Kim, H.; Sohn, A.; Yeo, I.; Kim, Y. Cost-Effective Automated Preparation of Serum Samples for Reproducible Quantitative Clinical Proteomics. J. Proteome Res. 2019, 18, 23372345.

(11) Wiśniewski, J. R.; Zougman, A.; Nagaraj, N.; Mann, M. Universal sample preparation method for proteome analysis. Nat. Methods 2009, 6, 359-362.

(12) Arul, A.-B.; Byambadorj, M.; Han, N.-Y.; Park, J. M.; Lee, H. Development of an Automated, High-throughput Sample Preparation Protocol for Proteomics Analysis. Bull. Korean Chem. Soc. 2015, 36, 1791-1798

(13) Specht, H.; Harmange, G.; Perlman, D. H.; Emmott, E.; Niziolek, Z.; Budnik, B.; Slavov, N. Automated Sample Preparation for High-Throughput Single-Cell Proteomics. BioRxiv 2018.

(14) Potriquet, J.; Laohaviroj, M.; Bethony, J. M.; Mulvenna, J. A modified FASP protocol for high-throughput preparation of protein samples for mass spectrometry. PLoS One 2017, 12, No. e0175967.

(15) Berger, S. T.; Ahmed, S.; Muntel, J.; Cuevas Polo, N.; Bachur, R.; Kentsis, A.; Steen, J.; Steen, H. MStern Blotting-High Throughput Polyvinylidene Fluoride (PVDF) Membrane-Based Proteomic Sample Preparation for 96-Well Plates. Mol. Cell. Proteomics 2015, 14, 28142823.

(16) Kulak, N. A.; Pichler, G.; Paron, I.; Nagaraj, N.; Mann, M. Minimal, encapsulated proteomic-sample processing applied to copynumber estimation in eukaryotic cells. Nat. Methods 2014, 11, 319324

(17) Batth, T. S.; Singh, P.; Ramakrishnan, V. R.; Sousa, M. M. L.; Chan, L. J. G.; Tran, H. M.; Luning, E. G.; Pan, E. H. Y.; Vuu, K. M.; Keasling, J. D.; et al. A targeted proteomics toolkit for highthroughput absolute quantification of Escherichia coli proteins. Metab. Eng. 2014, 26, 48-56.

(18) Chen, Y.; Vu, J.; Thompson, M. G.; Sharpless, W. A.; Chan, L. J. G.; Gin, J. W.; Keasling, J. D.; Adams, P. D.; Petzold, C. J. A rapid methods development workflow for high-throughput quantitative proteomic applications. PLoS One 2019, 14, No. e0211582. 
(19) Wessel, D.; Flügge, U. I. A method for the quantitative recovery of protein in dilute solution in the presence of detergents and lipids. Anal. Biochem. 1984, 138, 141-143.

(20) Weaver, L. J.; Sousa, M. M. L.; Wang, G.; Baidoo, E.; Petzold, C. J.; Keasling, J. D. A kinetic-based approach to understanding heterologous mevalonate pathway function inE. coli. Biotechnol. Bioeng. 2015, 112, 111-119.

(21) Bourmaud, A.; Gallien, S.; Domon, B. A quality control of proteomic experiments based on multiple isotopologous internal standards. EuPA Open Proteom. 2015, 8, 16-21.

(22) Mohammed, Y.; Percy, A. J.; Chambers, A. G.; Borchers, C. H. Qualis-SIS: automated standard curve generation and quality assessment for multiplexed targeted quantitative proteomic experiments with labeled standards. J. Proteome Res. 2015, 14, 1137-1146.

(23) Pino, L. K.; Searle, B. C.; Huang, E. L.; Noble, W. S.; Hoofnagle, A. N.; MacCoss, M. J. Calibration Using a Single-Point External Reference Material Harmonizes Quantitative Mass Spectrometry Proteomics Data between Platforms and Laboratories. Anal. Chem. 2018, 90, 13112-13117.

(24) Carr, S. A.; Abbatiello, S. E.; Ackermann, B. L.; Borchers, C.; Domon, B.; Deutsch, E. W.; Grant, R. P.; Hoofnagle, A. N.; Hüttenhain, R.; Koomen, J. M.; et al. Targeted peptide measurements in biology and medicine: best practices for mass spectrometry-based assay development using a fit-for-purpose approach. Mol. Cell. Proteomics 2014, 13, 907-917.

(25) Kensy, F.; Engelbrecht, C.; Büchs, J. Scale-up from microtiter plate to laboratory fermenter: evaluation by online monitoring techniques of growth and protein expression in Escherichia coli and Hansenula polymorpha fermentations. Microb. Cell Fact. 2009, 8, 68.

(26) Funke, M.; Buchenauer, A.; Mokwa, W.; Kluge, S.; Hein, L.; Müller, C.; Kensy, F.; Büchs, J. Bioprocess control in microscale: scalable fermentations in disposable and user-friendly microfluidic systems. Microb. Cell Fact. 2010, 9, 86.

(27) Kensy, F.; Zang, E.; Faulhammer, C.; Tan, R.-K.; Büchs, J. Validation of a high-throughput fermentation system based on online monitoring of biomass and fluorescence in continuously shaken microtiter plates. Microb. Cell Fact. 2009, 8, 31.

(28) Huang, D. W.; Sherman, B. T.; Lempicki, R. A. Systematic and integrative analysis of large gene lists using DAVID bioinformatics resources. Nat. Protoc. 2009, 4, 44-57. 